Article

\title{
Adsorption Behavior of Methyl Laurate and Dodecane on the Sub-Bituminous Coal Surface: Molecular Dynamics Simulation and Experimental Study
}

\author{
He Zhang ${ }^{\circledR}$, Wenli Liu *, Hongxiang $X u^{*} \mathbb{C}$, Qiming Zhuo® and Xiaopeng Sun \\ School of Chemical and Environmental Engineering, China University of Mining and Technology (Beijing), \\ Beijing 10083, China; zhe9127@163.com (H.Z.); zhuoqiming92@126.com (Q.Z.); xiaopeng201210@126.com (X.S.) \\ * Correspondence: liuwenli08@163.com (W.L.); xuhongxiang001@cumtb.edu.cn (H.X.); \\ Tel.: +86-010-6233-9883 (W.L.); +86-010-6233-9338 (H.X.)
}

Received: 12 December 2018; Accepted: 8 January 2019; Published: 9 January 2019

\begin{abstract}
Biodiesel was found to be a more effective collector on low-rank coal flotation than the common oily collectors (diesel and kerosene) in previous research. However, the micro-adsorption behavior of these collectors on the coal surface remains to be further explored. In the present work, the adsorption behavior of methyl laurate and dodecane, representing biodiesel and common oily collectors, was investigated using experimental and molecular dynamics (MD) simulation methods. The results of MD simulations showed that dodecane was difficult to diffuse on the surface of sub-bituminous coal and formed a spherical structure on the surface of sub-bituminous coal. Methyl laurate was adsorbed on the surface of coal via the head group (ester group), while the tail group (alkyl chain) was exposed to a liquid environment, forming a wider network structure on the coal surface. The above results, mainly attributed to methyl laurate, had a higher interaction with the sub-bituminous surface compared to dodecane. The self-diffusion coefficient results showed that the aggregate configurations of methyl laurate cause higher water mobility, which was more conducive to enhancing the hydrophobicity of the coal surface. The adhesion efficiency measurement and X-ray photoelectron spectrometer (XPS) analysis confirmed that methyl laurate could cover more oxygen-containing functional groups on the coal surface than dodecane, thus enhancing the hydrophobicity of coal. The results of simulations conformed to the experimental results.
\end{abstract}

Keywords: flotation; low-rank coal; adhesion efficiency; molecular dynamics; biodiesel

\section{Introduction}

Low-rank coals (lignite and sub bituminous coal), which account for more than half of the world's proven coal reserves, are a premium fuel and industrial raw material with high reactivity [1,2]. The processing and utilization of low-rank coal is of great help to the clean and efficient utilization of coal. Flotation is an effective means to upgrade fine coals, depending on the natural surface hydrophobicity of coal [3]. Low-rank coals are difficult to float using common oily collectors, such as kerosene and diesel, due to the oxidized surface with reduced hydrophobicity. Therefore, the selection of efficient collectors for low-rank coals has been the focus of attention $[4,5]$. Previous studies have found that introducing different oxygen-containing functional groups, such as hydroxyl groups and ether bonds, into low-rank coal collectors can effectively improve the collecting capacity of low-rank coal [6-9]. However, the collectors containing these functional groups are mostly petroleum products or chemical by-products. Due to the nonrenewable nature of fossil fuels, the use of these collectors will cause the waste of fossil fuels, while also being harmful to the environment. 
Biodiesel, composed of fatty acid methyl ester, is a biodegradable, renewable and nontoxic fuel, which can be used as an efficient collector for fine low-rank coal flotation [10,11]. Xia et al. [12] studied the collecting ability of biodiesel and diesel on the surface of low-rank coals and oxidized coals through a flotation experiment, revealing that the collection capability of biodiesel collectors was superior to that of diesel collectors. The floatability of low-rank coals is enhanced by the increase of coal surface hydrophobicity through the ester groups of fatty acid methyl esters in biodiesel bonded to the oxygen sites on the coal surface. Although it has been determined that fatty acid methyl esters with ester groups have a better collecting performance than common nonpolar hydrocarbon collectors for low-rank coal, the mechanism of their action is less involved, explaining the effect of the ester group on the collecting performance of fatty acid methyl esters from a microscopic level.

Molecular dynamics simulation is a powerful tool to reveal the interaction mechanism between collectors and minerals at microscopic level [13-16]. The adsorption behavior of collectors on mineral surfaces in an aqueous environment can be obtained from the atomic or molecular level by molecular dynamics simulation. Li et al. [17] used molecular dynamics (MD) simulations to study the adsorption behavior of sodium oleate on siderite, hematite, and quartz in an aqueous environment. The results showed that sodium oleate can effectively collect siderite at neutral $\mathrm{pH}$. Zhang et al. [18] studied the adsorption behavior of dodecylamine, butanol, hexanol, and octanol on the surface of magnesite and dolomite using the molecular dynamics method. The simulation results showed that monohydric alcohol can enhance the adsorption of dodecylamine on mineral surfaces. Zhang et al. [19] adopted molecular dynamics simulations to describe the adsorption of three collectors (dodecane, nonylbenzene, and nonylphenol) on the surface of Wiser bituminous coal. It was found that the functional group type of collectors influenced the interactions between collector molecules and coal surfaces. Dodecane with a non-polar hydrocarbon structure has the most significant effect on the bituminous coal collecting performance. Guo et al. [20] studied the adsorption of n-dodecyl $\beta$-D-maltoside $\left(C_{12} G_{2}\right)$ and dodecyl hepta glycol $\left(\mathrm{C}_{12} \mathrm{E}_{7}\right)$ molecules on the surface of Wender lignite using the molecular dynamics simulation. It was found that the $\mathrm{C}_{12} \mathrm{E}_{7}$ with $-\mathrm{CH}_{2}-\mathrm{O}-\mathrm{CH}_{2}$ is a better reagent than $\mathrm{C}_{12} \mathrm{G}_{2}$ with $-\mathrm{OH}$ for the lignite.

In summary of the above studies, it can be seen that molecular dynamics simulation has attracted great attention in attempts to reveal the mechanism of collectors and minerals at the microlevel, but few studies have focused on the micro-adsorption behavior of the environment-friendly collector, fatty acid methyl ester, on the surface of low-rank coal.

In this paper, the fatty acid methyl esters with 12 carbons (methyl laurate) and the nonpolar hydrocarbon with the same carbons (dodecane) were selected to represent biodiesel and common oily collectors. The adsorption behavior of two collectors on the surface of low-rank coal was studied using the molecular dynamics method. The water/collectors/coal system was established to quantitatively analyze the molecular scale structure, dynamics, and energy behavior of the system. The experiment was also performed to verify the rationality of the simulation.

\section{Materials and Methods}

\subsection{Materials and Reagents}

The sub-bituminous coal samples were obtained from the Bulianta Coal Mine (Erdos, Inner Mongolia, China). The proximate and ultimate analysis of the coal sample was conducted, as shown in Table 1. 
Table 1. The proximate and ultimate analysis of the coal sample ${ }^{1}$.

\begin{tabular}{cccccccc}
\hline \multicolumn{3}{c}{ Proximate Analysis $(\mathbf{w t} \%)$} & \multicolumn{4}{c}{ Ultimate Analysis } & $\left(\mathbf{w t}_{\text {daf }} \%\right)$ \\
\hline $\mathbf{M}_{\mathrm{ad}}$ & $\mathbf{A}_{\mathrm{ad}}$ & $\mathbf{V}_{\text {daf }}$ & $\mathbf{C}$ & $\mathbf{H}$ & $\mathbf{O}^{\mathbf{2}}$ & $\mathbf{N}$ & $\mathbf{S}$ \\
\hline 5.84 & 10.46 & 31.26 & 74.33 & 5.23 & 18.97 & 1.03 & 0.44 \\
\hline
\end{tabular}

${ }^{1} \mathrm{M}_{\mathrm{ad}}$ is moisture mass fraction of samples on air-dried basis; $\mathrm{A}_{\mathrm{ad}}$ is ash mass fraction of samples on a dry basis; $\mathrm{V}_{\mathrm{daf}}$ is volatile matter mass fraction of samples on a dry and ash-free basis; and $\mathrm{wt}_{\mathrm{daf}} \%$ is weight percentage of various elements on a dry and ash-free basis. ${ }^{2}$ By difference.

Based on Table 1, the oxygen content in coal samples is as high as $18.97 \%$, which indicates that the surface of the coal sample was abundant with oxygen content. The coal samples with a density of $1.3-1.4 \mathrm{~g} / \mathrm{cm}^{3}$ were obtained by a fine float-sink test to exclude the influence of ash content on subsequent experiments. For the attachment efficiency measurement, the coal samples were sieved to obtain the particle size in the range of $0.10-0.15 \mathrm{~mm}$. In addition, the sample was crushed to $-74 \mu \mathrm{m}$ for the X-ray photoelectron spectrometer (XPS) experiment. In order to obtain modified coal samples after the adsorption of collectors, the coal pulp and the collectors were added to a cell under stirring at a speed of $1800 \mathrm{r} / \mathrm{min}$ for $2 \mathrm{~min}$. The pulp concentration was $80 \mathrm{~g} / \mathrm{L}$. The dosage of collectors was $2 \mathrm{~kg}$ per ton of dry feed coal. The sample particles were filtered from the pulp and the obtained sample was dried in a vacuum drying oven at $105^{\circ} \mathrm{C}$ for $24 \mathrm{~h}$.

The methyl laurate (purity: 99\%; formula: $\mathrm{C}_{13} \mathrm{H}_{26} \mathrm{O}_{2}$ ), which represents biodiesel, was purchased from Shanghai Macklin Biochemical Co., Ltd., Shanghai, China. The dodecane (purity: 99\%; formula: $\mathrm{C}_{12} \mathrm{H}_{26}$ ), representing common oily collectors, was obtained from Shanghai Aladdin reagent Co., Ltd., Shanghai, China.

\subsection{Molecular Simulation Details}

The molecular dynamic simulations were conducted in Forcite modules using Material Studio 2018 software (Dassault Systemes Biovia K.K. Biovia, San Diego, CA, USA). The COMPASS II force field was applied for all the simulations, which extend the coverage of the COMPASS force field for polymer and drug-like compounds in the materials arena [21]. The water/collector/coal model, containing a sub-bituminous surface, a collector layer, and an aqueous layer, was constructed to investigate the interaction among them. The proposed Hatcher sub-bituminous model was selected to build the sub-bituminous coal surface [22]. The SPC (simple point charge) water model was selected in this paper. The molecular structures of the Hatcher model, water, dodecane, and methyl laurate are shown in Figure 1. The atomic charges for all molecules were automatically distributed through the COMPASS II force field, which was also shown in Figure 1. The sub-bituminous coal surface model consisted of 40 geometrically optimized sub-bituminous molecules, which were packed into a 3D cell of $45 \AA \times 45 \AA(\mathrm{X} \times \mathrm{Y})$ by the amorphous cell module (Figure 2). The annealing algorithm was conducted to achieve structure relaxation of the coal surface model. The initial temperature of the annealing algorithm increased from $298 \mathrm{~K}$ to $1098 \mathrm{~K}$ and finally returned to $298 \mathrm{~K}$. The temperature increased and decreased once every $50 \mathrm{~K}$ and 100 ps MD simulation was carried out at each temperature. The 20 collector molecules and 2000 water molecules were also packed into the 3D cell to form the collector layer and the water layer, respectively, with the same method. Then, a vacuum with a thickness of around $110 \AA$ was applied at the top of these layers to avoid any interaction between the top and bottom surface of the model because of the periodic boundary conditions.

The built model was optimized to obtain an energy minimization structure. Then, the MD simulation was carried out at $298 \mathrm{~K}$ using the constant-volume and -temperature (NVT) ensemble with 500 ps simulation time and $1 \mathrm{fs}$ time step. The van der Waals and long-range electrostatic interactions were calculated using the atom-based method and the Ewald summation method. The temperature control method was set to Nosé [23] thermostat. 
(a)

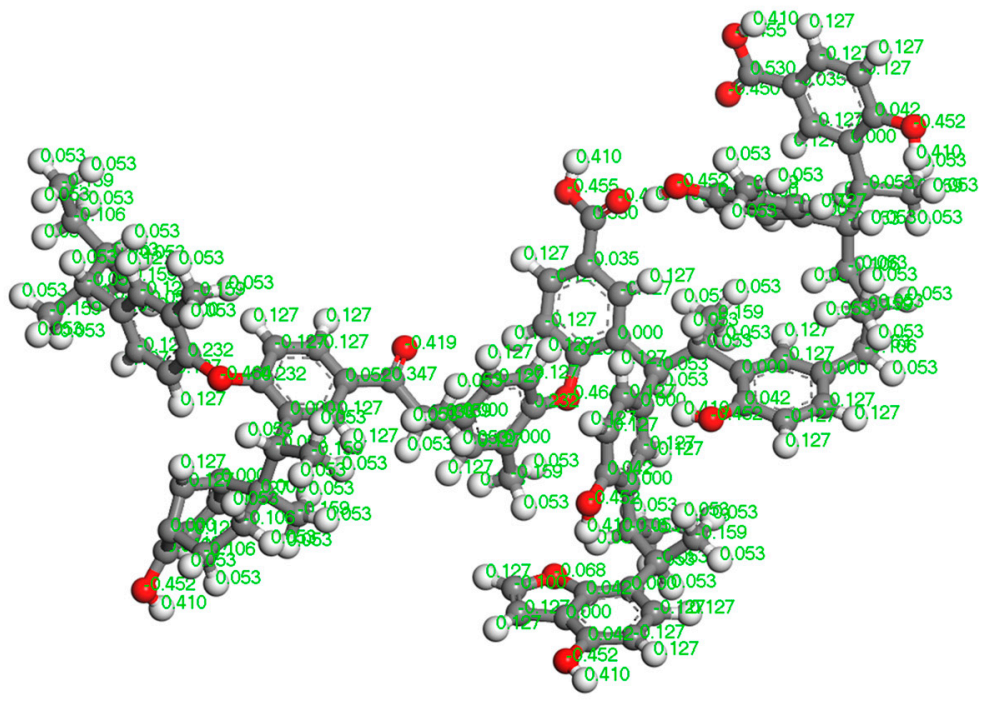

(b)

(c)
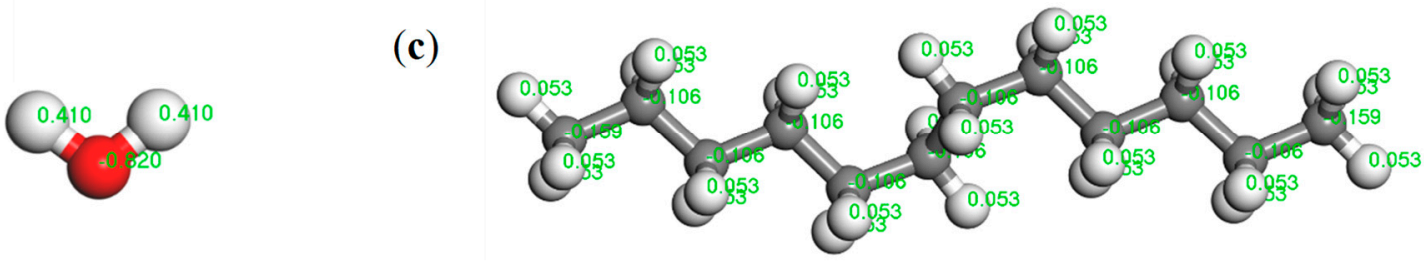

(d)

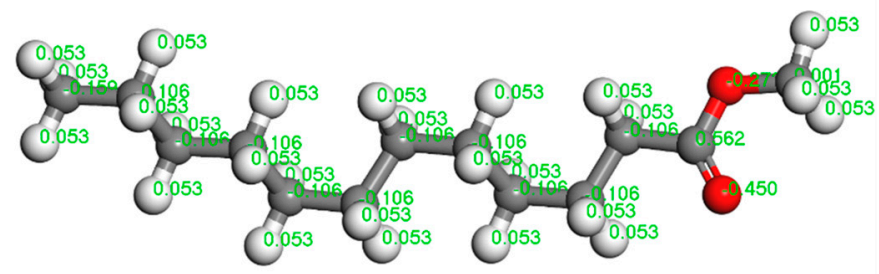

Figure 1. (a) Molecular model structure of Hatcher. (b) Water molecular structure. The structure of (c) dodecane and (d) methyl laurate. The representation of gray, white, and red is the $\mathrm{C}, \mathrm{H}$, and $\mathrm{O}$ atom, respectively.

(a)

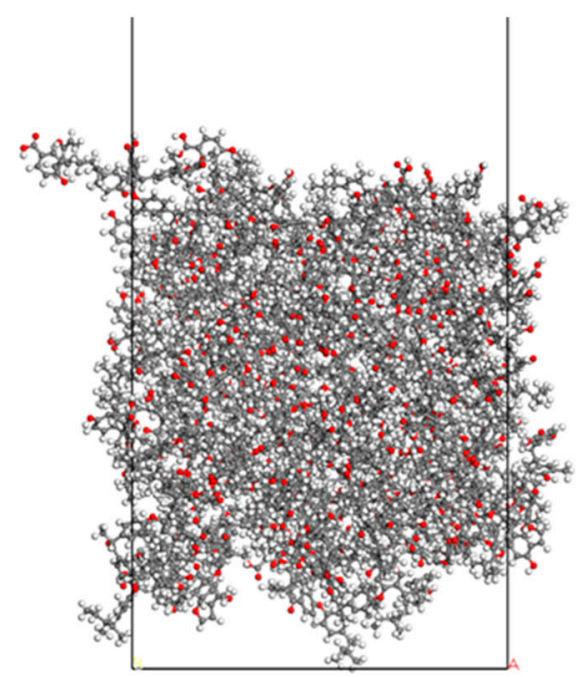

(b)

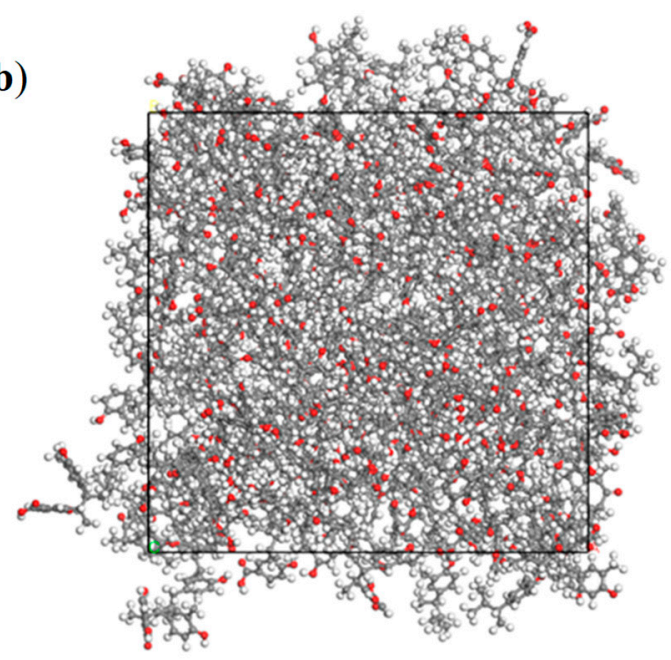

Figure 2. The surface model of low-rank coal. (a) The side view and (b) top view. The representation of gray, white, and red is the $\mathrm{C}, \mathrm{H}$, and $\mathrm{O}$ atom, respectively. 


\subsection{XPS}

The X-ray photoelectron spectrometer (XPS), Thermo Scientific Escalab 250Xi (Thermo Fisher Scientific, Waltham, MA, USA), with an AlK Alpha anode operating at $200 \mathrm{~W}$ was used. A pass energy of $100 \mathrm{eV}$ was applied for the spectrum of the survey scan with the step size of $1000 \mathrm{MeV}$. Before data analysis, $284.8 \mathrm{eV}$ was selected as the C1s spectral peak calibration standard for calibration [24,25].

\subsection{Attachment Efficiency Measurement}

The attachment efficiency between the coal particles and air bubbles was measured using a self-constructed attach efficiency instrument consisting of a funnel moving module, a bubble micro displacement device, a bubble generation and adjustment module, a feed funnel, an observation room, a camera, and an LED (Light-Emitting Diode) array light source [26]. The experiment was roughly divided into two steps. The first was to record the adhesion process between particles and bubbles in the observation room by camera. The second was to count the adhesion efficiency of particles at different collision angles using a program developed on the MATLAB (matrix \& laboratory) platform. In order to accurately represent the relative position of particles and bubbles, the position of the bubble center was located at the origin of coordinates, the horizontal direction was set at the $X$-axis, and the vertical direction was set at the $Z$-axis. When the distance between the particle and the bubble was less than 1 pixel, the coordinates of the particle pixels pointing closest to the edge of the bubble were determined as the collision point coordinates. The angle between the collision point and the vertical direction of the bubble center was defined as the collision angle, as shown in Figure 3.

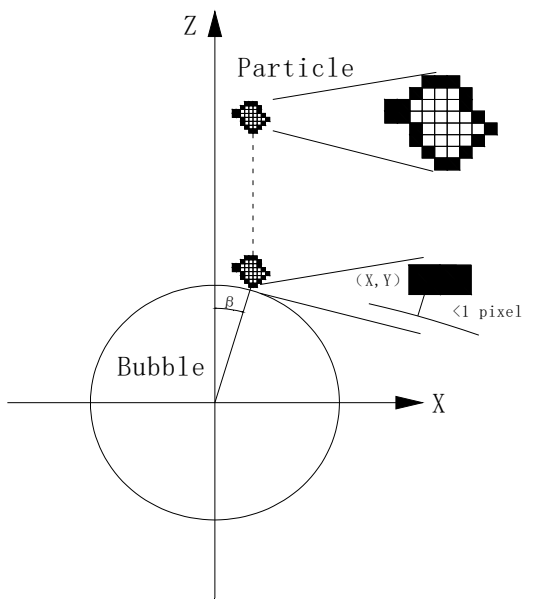

Figure 3. Sketch map of particle collision angle.

\section{Results and Discussion}

\subsection{Molecular Dynamics Simulation}

\subsubsection{Adsorption Structure of Collectors on the Coal/Water Interface}

The initial and equilibrium configurations of the water/collector/coal three-phase system model were obtained using the abovementioned molecular dynamics method, as shown in Figure 4 . From Figure 4, the collector is evenly distributed in the reagent layer in the initial state. With the simulation, the collector spontaneously aggregated at the coal-water interface and adsorbed to the coal surface, which meant that the adsorption of the collector will change the surface properties of coal and then affect the wetting effect of coal. 


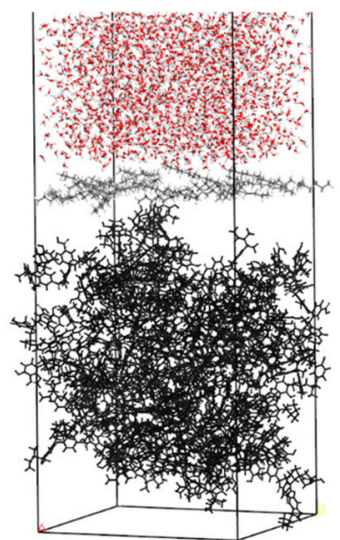

(a)
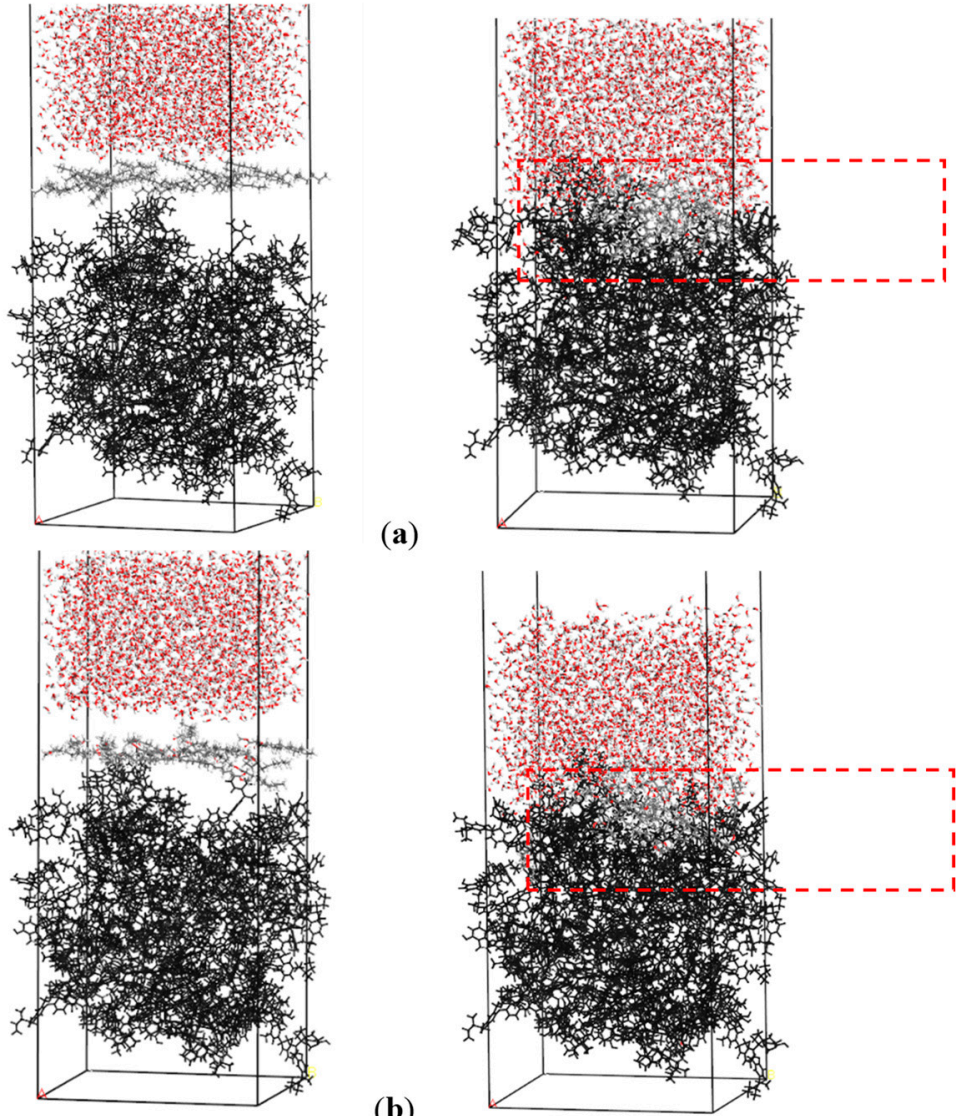

Figure 4. The initial (left) and equilibrium (right) adsorption configuration on the surface of sub-bituminous coal in aqueous environment: (a) water/dodecane/coal system and (b) water/methyl laurate/coal. For clarity, the coal surface models are shown as black.

The configurations of the two collectors in adsorption equilibrium are different. In order to observe the molecular morphology of collector adsorbed on coal surface at equilibrium, the reagent layer (red box) in Figure 4 is enlarged, as shown in Figure 5.

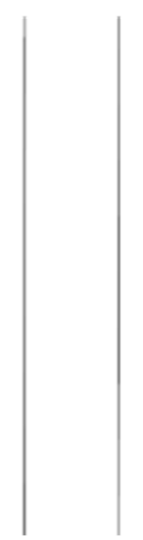

(a)

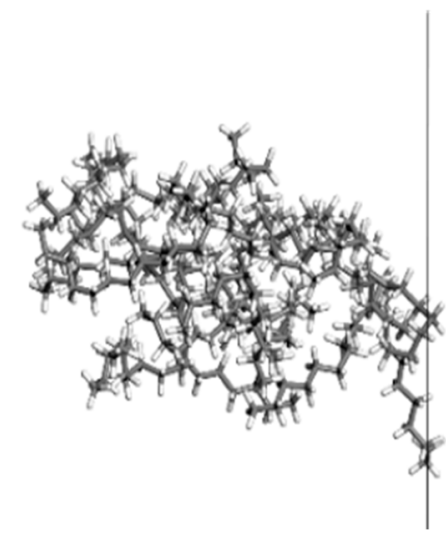

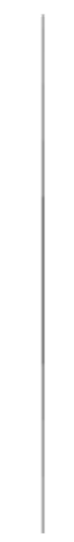

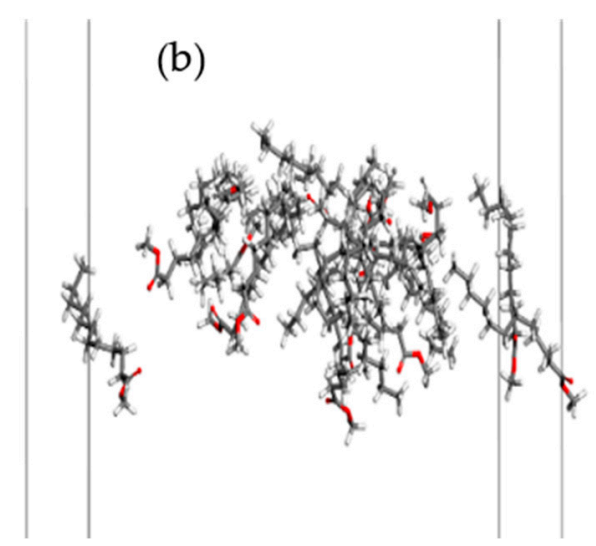

Figure 5. The equilibrium adsorption configuration of collectors on the surface of sub-bituminous coal in an aqueous environment: (a) dodecane and (b) methyl laurate. For clarity, the water molecules and coal surface models are not shown.

Figure 5 shows that dodecane molecules form a spherical structure on the surface of coal, while methyl laurate forms a network-like structure. The different configuration mentioned suggests 
that dodecane molecules are difficult to diffuse on the surface of sub-bituminous coal with abundant hydrophilic groups, while methyl laurate can diffuse easily on the surface of sub-bituminous coal.

The mass density distribution of coal molecules, collector molecules, and water molecules along the Z-axis was simulated to quantitatively characterize the molecular morphology and spatial position of dodecane and methyl laurate at the coal-water interface, as shown in Figure 6. The Z-axis is perpendicular to the coal surface, and the direction is vertical upward. The abscissa is the distance along the Z-axis, which is the distance to the bottom of the system. The ordinate is the density of the molecule in the $X \times Y$ plane (parallel to the coal surface), located at different positions of the Z-axis. By calculating the density distribution along the $Z$-axis, the degree of aggregation of molecules in the $\mathrm{X} \times \mathrm{Y}$ plane can be obtained at different positions of the Z-axis. Table 2 lists the distributions of coal molecules, collector molecules, and water molecules along the Z-axis in the three systems, so as to express more clearly the distributions of each molecular layer along the Z-axis in Figure 6.
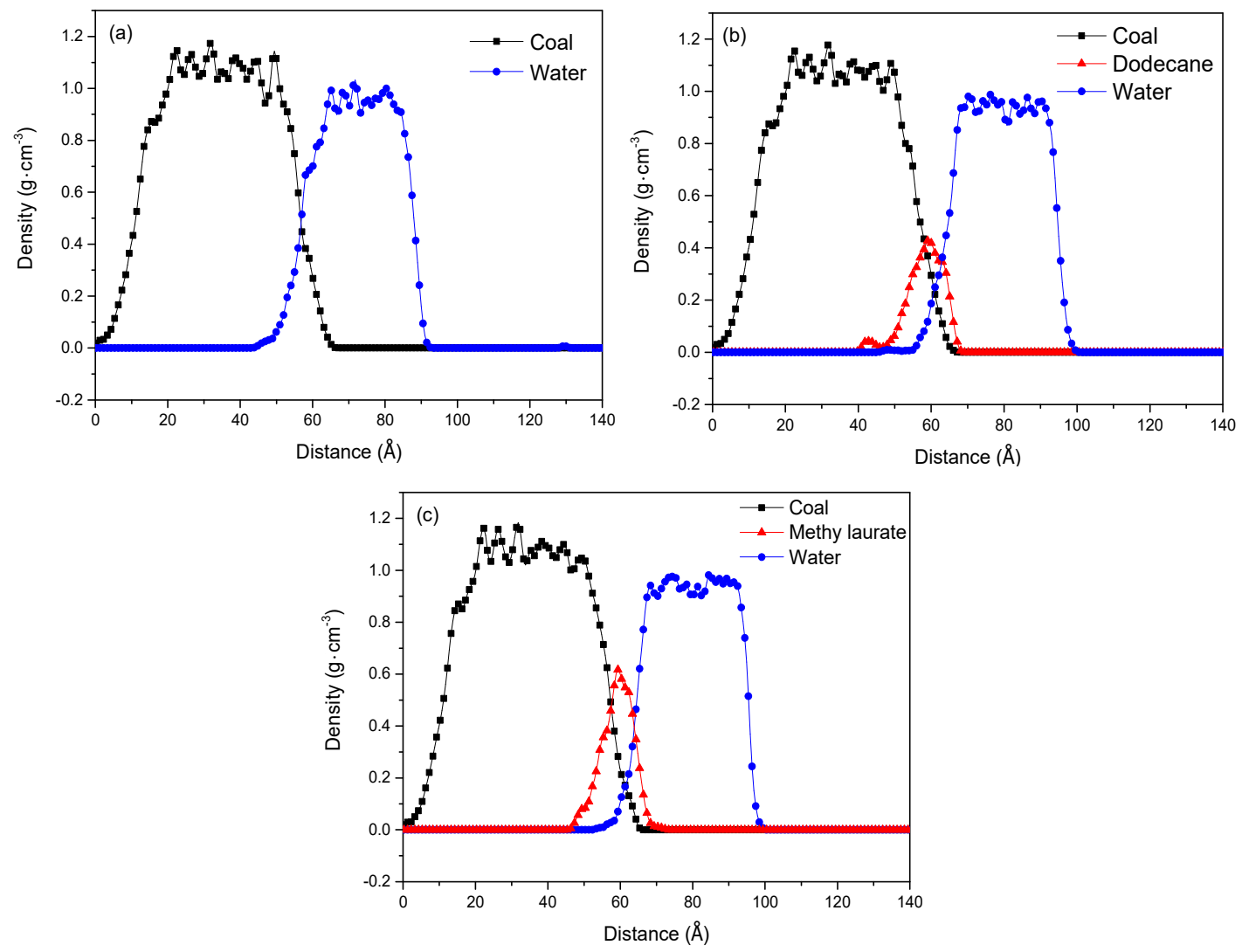

Figure 6. The Z-axis density distributions of water, collectors, and coal: (a) water/coal, (b) water/ dodecane/coal, and (c) water/methyl laurate/coal system.

Table 2. Scope of Z-axis distribution of water, collectors, and coal molecular layers in three systems $(\AA)$.

\begin{tabular}{cccc}
\hline System & Coal & Collectors & Water \\
\hline water/coal & $0-66.66$ & - & $43.34-93.02$ \\
water/dodecane/coal & $0-66.62$ & $39.32-70.16$ & $45.34-100.56$ \\
water/methyl laurate/coal & $0-66.59$ & $45.31-70.44$ & $52.34-100.42$ \\
\hline
\end{tabular}

From Figure 6 and Table 2, it can be seen that the adsorption process does not affect the density distribution of the coal molecule, and there is a consistent density distribution in the three systems. After the adsorption of collectors, the overlapped density distributions between the water and coal decreased. This meant that the collector molecules were adsorbed on the sub-bituminous coal to form an isolating layer, which can keep water away from the surface of sub-bituminous coal. Table 2 
shows the water layer mainly distributes in the range of $45.34-100.56 \AA$ in the water/dodecane/coal system, while in the water/methyl laurate/coal system, the water layer distributes in the range of 52.34-100.42 A. The results showed that the network configuration formed by methyl laurate can cover the coal surface more effectively than the spherical configuration of dodecane and prevent water molecules from contacting with coal.

From the density distribution of collectors along the Z-axis direction in Figure 6, it is shown that the distribution of collectors mainly exists at the coal-water interface, and the density distribution between coal and collectors had an apparent overlap. This does not imply that the collector molecules penetrated the coal surface, but because the coal surface has a rough surface, the coverage of collector molecules to the microscopic valleys on the coal surface leads to the overlap of the collectors and the coal surface. By comparing the density distributions of the two collectors along the Z-axis, it was found the density peaks of both collectors appeared around $60 \AA$, but the peak intensity of methyl laurate was obviously higher than that of dodecane. The density distribution of methyl laurate on the Z-axis is mainly in the range of $45-70 \AA$, which is less than that of dodecane in the range of $40-70 \AA$. The above results imply that the density distributions of methyl laurate are concentrated in a smaller $Z$-axis range than those of dodecane. In other words, when the dosage of the two collectors is the same, the molecule of methyl laurate has a wider distribution on the $\mathrm{X} \times \mathrm{Y}$ plane perpendicular to the $Z$-axis (parallel to the coal surface), which indicates that methyl laurate is easier to diffuse on the coal surface than dodecane and has a wider coverage on the coal surface.

The methyl laurate molecule is a polar molecule with a head group (ester group) and tail group (fat chain). The orientation of the methyl laurate molecule covering the coal surface has an important influence on the hydrophobicity of the coal surface. The Z-dependent mass density for the head group (ester group) and tail group (fatty chain) is calculated to determine the adsorption configuration in the water-coal interface at the initial and final time (Figure 7). At the initial state, there is no difference between the Z-dependent mass density of the head group and tail group. When the adsorption was completed, reorientation occurred in the collector molecules. The head group (ester group) of the methyl laurate molecules is directed toward the surface of coal through hydrogen bonds, while the tail group (fatty chain) is far away from the coal surface due to hydrophobic action. The ester group of the collector molecules covered the hydrophilic sites on the coal surface and the fatty chain of the collector was exposed to the aqueous phase, which made the coal surface more hydrophobic.
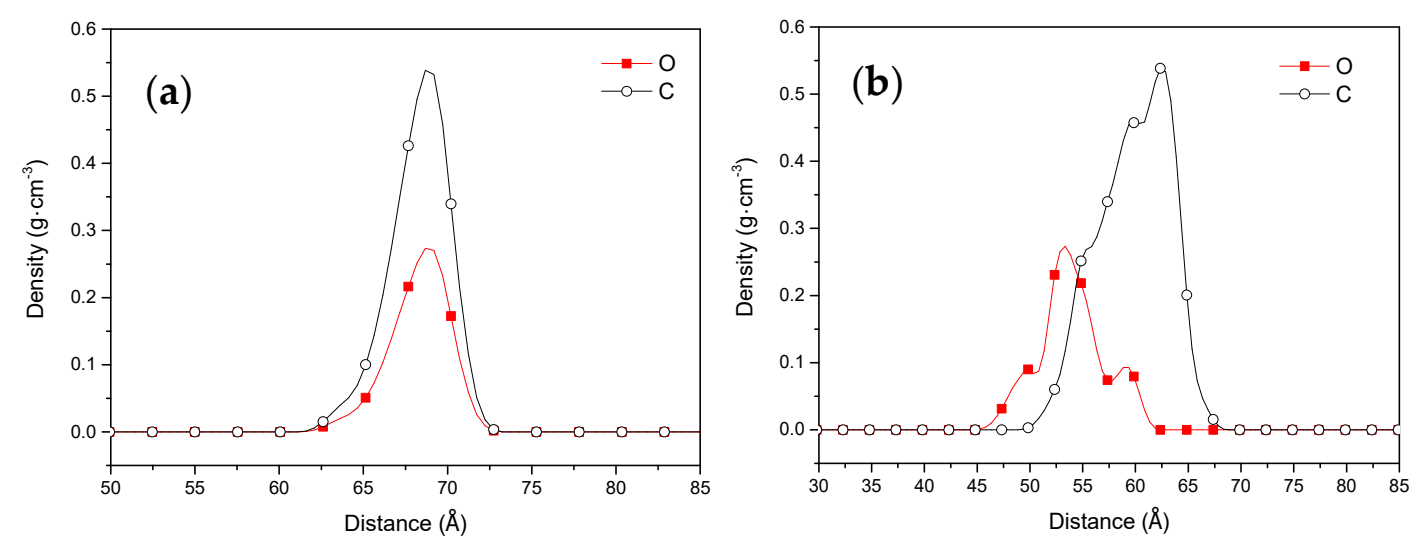

Figure 7. The Z-axis mass density for the head group (ester group) and tail group (fatty chain): (a) the initial structure and $(b)$ the equilibrium structure.

\subsubsection{Interaction Energies between Collectors and Coal}

The difference in the adsorption behavior of the two collectors on the coal surface is mainly attributed to the difference of interaction strength between the two collectors and the sub-bituminous coal surface. In order to quantitatively measure the interaction strength between collectors and the sub-bituminous coal surface, the interaction energies between collectors and the surface of 
sub-bituminous coal was calculated. The more negative the binding energy between collectors and sub-bituminous coal was, the more conducive it was to the adsorption of collectors on the coal surface. The interaction energy between the collector and the coal surface can be determined by the following formula [27]:

$$
\operatorname{Int}_{c \& s}=\left(E_{t}-E_{c}-E_{s+w}-E_{s}-E_{w+c}+E_{w}+E_{c+s}\right) / 2
$$

where Int $t_{c \& s}$ is the interaction energy between collectors and the coal surface, $E_{t}$ is the total energy of the system, and $E_{c}, E_{s}$, and $E_{w}$ are the energy of collectors, coal surface, and water, respectively. $E_{s+w}$, $E_{w+c}$, and $E_{c+s}$ represent the energy of water/coal surface, water/collectors, and collectors/coal surface models, respectively.

The interaction energies ( $I n t_{\mathrm{c \& s}}$ ) in the presence of methyl laurate and dodecane are -365.40 and $-260.97 \mathrm{~kJ} / \mathrm{mol}$, respectively. The results showed that the interaction between coal and dodecane is relatively small, which also means that aliphatic hydrocarbons are not easily adsorbed on the surface of coal with more oxygen-containing groups but are aggregated into spherical structures under the influence of intermolecular hydrophobic force. The interaction between methyl laurate and coal is stronger because of the strong hydrogen bond between ester groups in methyl laurate and oxygen-containing functional groups on the surface of sub-bituminous coal. This stronger interaction force will be conducive to the diffusion of methyl laurate on the coal surface and the formation of stable adsorption between methyl laurate and coal surface through ester groups.

\subsubsection{Mobility of Water Molecules before and after the Adsorption of Collectors}

The adsorption of collectors on the sub-bituminous coal surface will affect the diffusion behavior of water molecules on the coal surface, which can be reflected by the mean square displacement (MSD) and the self-diffusion coefficient $(D)$. MSD can quantify the diffusion strength of the water molecule on the coal surface with time [28-30]:

$$
M S D=\frac{1}{N} \sum_{i=1}^{N}\left(r_{i}(t)-r_{i}(0)\right)^{2}
$$

Here, $N$ is the number of water molecules, $t$ is the statistical time, and $r_{i}(t)$ and $r_{i}(0)$ represent the position of the center of water molecular $i$ at the time $t$ and the initial time, respectively.

The MSDs of water molecules on (modified) coal surfaces are obtained by molecular dynamics simulation, as shown in Figure 8. As can be seen from Figure 8, the diffusion strength of water molecules on the surface of (modified) coal increases gradually with the extension of simulation time, and the enhancement range follows that of methyl laurate modified coal > dodecane modified coal $>$ unmodified coal.

The self-diffusion coefficient $(D)$, which represents the physical quantity of the water molecule diffusion degree on the coal surface, can reflect the diffusion ability of the water molecule on the coal surface. The high mobility was conducive to the displacement of water molecules from the (modified) coal surface, which made the bubbles easier to attach on the coal surface. The self-diffusion coefficient $(D)$ can be calculated by the Einstein equation:

$$
D=\frac{1}{6} \lim _{t \rightarrow \infty} \frac{d}{d t}(M S D)=\frac{1}{6} K_{M S D}
$$

Here, $K_{\mathrm{MSD}}$ is the slope of the MSD curve.

According to Equation (3), the self-diffusion coefficient $(D)$ of water in water/coal and water/collector/coal systems can be determined, as shown in Table 3. Table 3 shows there is the lowest water molecular diffusion coefficient in the water/coal system. This implies that the diffusion of water molecules on the surface of sub-bituminous coal is limited in the water/coal system. This limitation is due to the existence of a large number of polar groups on the surface of sub-bituminous coal, which have a strong hydrogen bonding effect on polar water molecules, thus making the water 
molecules adsorb tightly on the surface of sub-bituminous coal. In the water/collector/coal system, the diffusion degree of water molecules is more intense, which is mainly due to the hydrophobic effect of the alkyl chain in the collector, which repels water molecules and inhibits the adsorption of water molecules on the surface of sub-bituminous coal. Among the two collectors with the same carbon number of nonpolar hydrocarbons, the methyl laurate containing ester group can more effectively reduce the polar groups on the coal surface and promote the diffusion of water molecules on the coal surface.

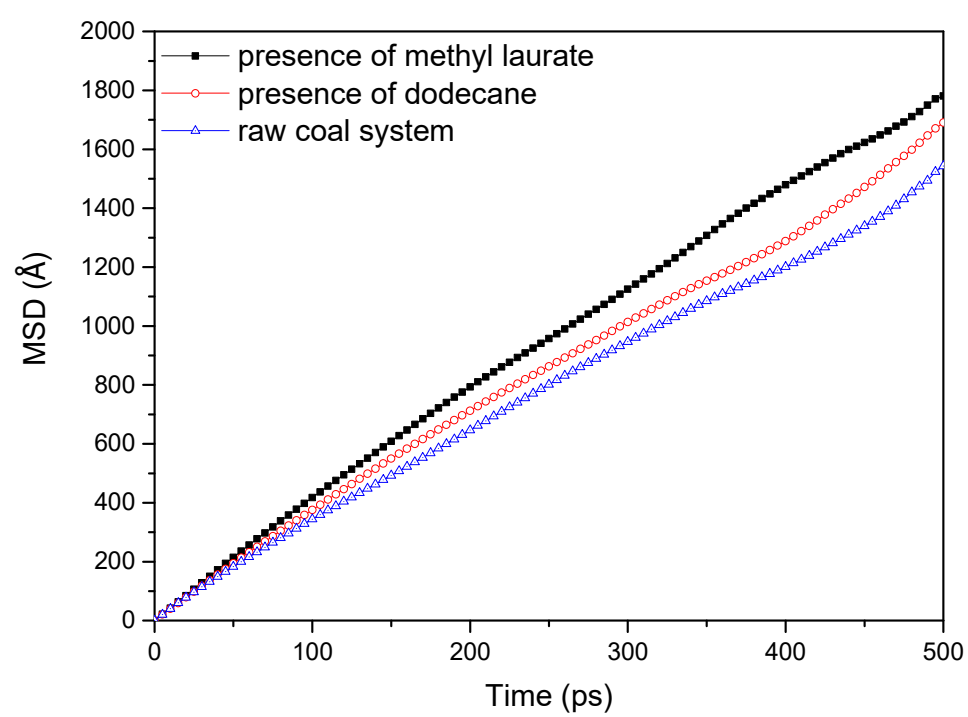

Figure 8. Mean square displacement $(M S D)$ curves of water molecules at the absence and presence of collectors.

Table 3. The self-diffusion coefficient $(D)$ of water in the water/coal and water/collector/coal system.

\begin{tabular}{|c|c|}
\hline System & $D\left(10^{-9} \mathrm{~m}^{2} / \mathrm{s}\right)$ \\
\hline water/coal & 4.90 \\
\hline water/dodecane/coal & 5.32 \\
\hline water/methyl laurate/coal & 5.88 \\
\hline
\end{tabular}

\subsection{Analysis of Experimental Results}

The adsorption behavior of two collectors on sub-bituminous coal surface is determined by the abovementioned molecular dynamics simulation. The results show that the methyl laurate with ester group can more effectively cover the oxygen-containing functional groups on coal surface than dodecane and effectively promote the diffusion of water molecules on the coal surface. In order to verify the simulation results from a macro perspective, the XPS method was used to determine the changes of oxygen functional groups on the coal surface before and after adsorption by different collectors. The hydrophobic change of the coal surface under different collectors was determined by measuring the adhesion efficiency between coal and air bubbles.

\subsubsection{XPS Analysis}

X-ray photoelectron spectroscopy (XPS) is an effective surface analysis method, which can provide the element compositions and their relative contents on the surface of coal samples [25]. XPS C1s and O1s spectra are presented in Figures 9 and 10. 

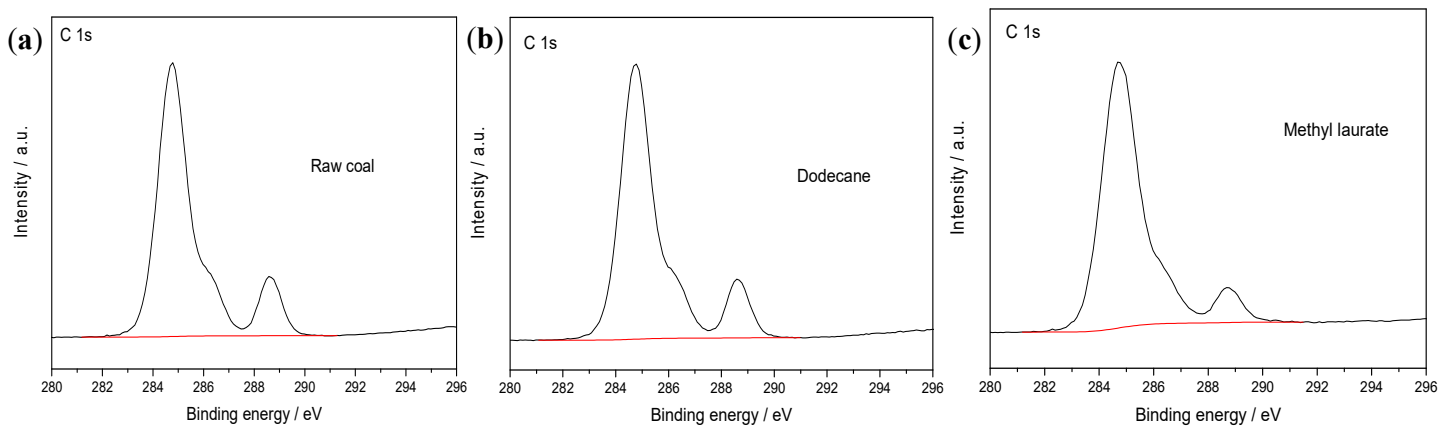

Figure 9. X-ray photoelectron spectroscopy (XPS) C1s spectra of (a) raw coal, in the presence of (b) dodecane, and (c) methyl laurate.
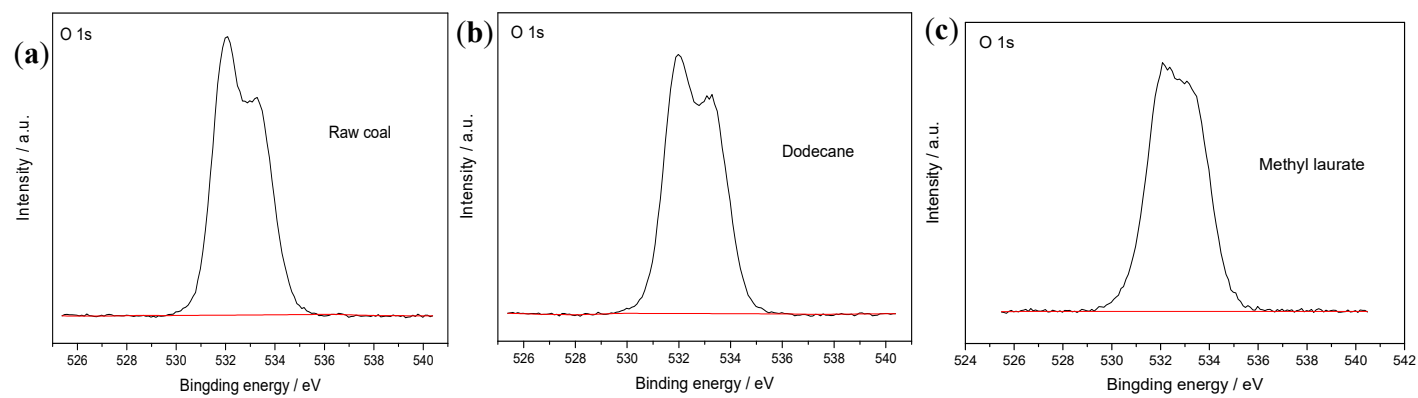

Figure 10. XPS O1s spectra of (a) raw coal, in the presence of (b) dodecane, and (c) methyl laurate.

From Figures 9 and 10, it can be seen that the intensity of the C1s and O1s peaks on the coal surface does not change significantly after the adsorption of dodecane. After the addition of methyl laurate, the intensity of the $\mathrm{C} 1$ s peak obviously increases, while the intensity of O1s peak is weakened. In order to quantitatively describe the change of peak intensity on the coal surface, the sum of the atomic $\mathrm{C} 1 \mathrm{~s}$ and $\mathrm{O} 1 \mathrm{~s}$ content was set to $100 \%$, and the atomic percentage of two elements is shown in Table 4.

Table 4 shows that the content of carbon increases slightly after the adsorption of dodecane, while the content of oxygen decreases slightly. The ratio of oxygen to carbon atoms also changes little, which is from $29.65 \%$ to $26.41 \%$. The main reason is that it is difficult for dodecane to diffuse effectively on the surface of coal. Although dodecane has a certain coverage effect on the oxygen-containing functional groups on the surface of coal, its effect is not obvious. After methyl laurate adsorption, the carbon content on coal surface increased from $77.13 \%$ to $84.16 \%$, while the oxygen content decreased from $22.87 \%$ to $15.84 \%$. The ratio of oxygen to carbon atoms is much lower than that of coal surface modified by dodecane. This result shows that methyl laurate can cover the hydrophilic sites of sub-bituminous coal more effectively and reduce the number of oxygen functional groups on the coal surface. This is consistent with the simulation results.

Table 4. The composition of $\mathrm{C}$ and $\mathrm{O}$ on the (modified) coal surface $(\mathrm{C}+\mathrm{O}=100 \%)$.

\begin{tabular}{cccc}
\hline Element & Raw Coal & Dodecane & Methyl Laurate \\
\hline C & 77.13 & 79.11 & 84.16 \\
O & 22.87 & 20.89 & 15.84 \\
O/C & 29.65 & 26.41 & 18.82 \\
\hline
\end{tabular}

\subsubsection{Attachment Efficiency Measurement}

The adhesion between coal particles and bubbles is the key to the flotation process, which is mainly affected by the hydrophobicity of the coal surface [31-33]. The higher adsorption efficiency indicates that the coal surface has better hydrophobicity. Adhesion efficiency is defined as the ratio of 
the number of particles adhering to a bubble at a given location to the total number of particles at that location, which can be expressed as follows:

$$
E=\frac{N_{a}}{N_{t}}
$$

where $E$ is the attachment efficiency between coal particles and bubbles, $N_{a}$ is the number of coal particles for adhesion, and $N_{t}$ is the total number of particles. The relationship between coal sample adhesion efficiency and collision angle is shown in Figure 11.

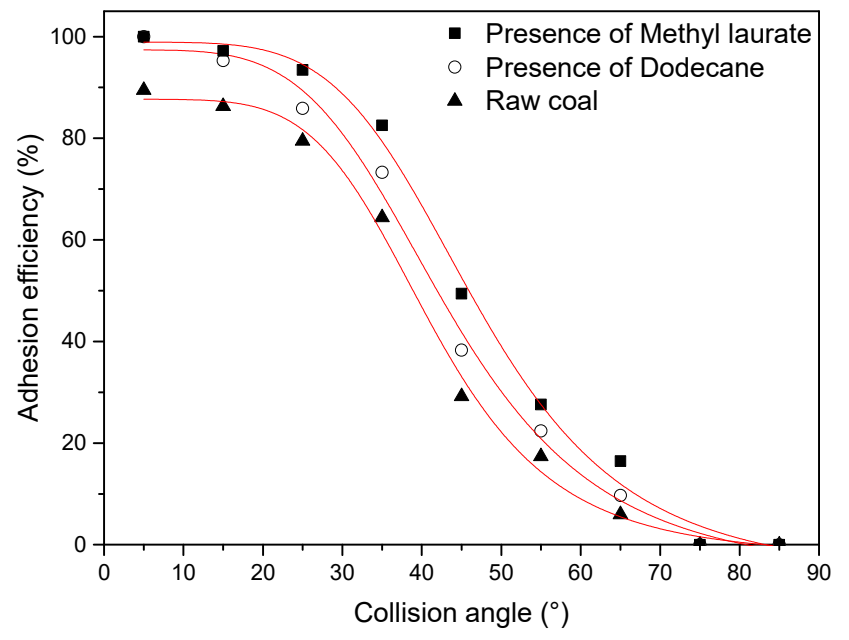

Figure 11. Adhesion efficiency of coal samples.

The adhesion efficiencies of 1000 effective particles at different positions of bubbles are counted to ensure the accuracy of the experiment. To quantify the horizontal position of particles relative to bubbles, the collision angle is chosen as the $X$-axis. The $Y$-axis is adhesion efficiency.

From Figure 11, the attachment efficiency between raw coal and bubble is the lowest, which indicates that the raw coal has poor hydrophobicity. This result is mainly attributed to the strong interaction between water molecules and the abundant oxygen-containing functional groups on the surface of raw coal, which prevented the coal particles from adhering bubbles. With the addition of collectors, the adhesion efficiency of the two collectors is improved, and the adhesion efficiency of coal particles adsorbed by methyl laurate is higher than that of dodecane at different positions of bubbles. This meant that the adsorption of collectors can improve the hydrophobicity of the coal surface and the addition of methyl laurate has a better hydrophobicity performance on the modified coal surface compared to dodecane. According to the simulation results, the main reason for this phenomenon is that the abundant hydrophilic sites limit the diffusion of dodecane on the coal surface, resulting in a large of number of oxygen-containing functional groups on the coal surface still exposed to an aqueous environment. It is easier to cover the hydrophilic part of the coal surface with the ester group of methyl laurate, while the aliphatic hydrocarbon is oriented towards the water environment and enhances hydrophobicity.

\section{Conclusions}

Methyl laurate and dodecane with the same carbon number of nonpolar hydrocarbons were selected to determine the effect of ester groups in methyl laurate on the collecting performance of low-rank coal. The adsorption behavior of two collectors on coal surface was studied by means of experiments and computer simulation.

(1) The adsorption behavior of two collectors was studied using molecular dynamics (MD) simulation. The results showed that the two collectors adsorbed on the interface between coal and water to form an isolating layer on water. It is difficult for dodecane to diffuse on the 
surface of sub-bituminous coal containing a large number of oxygen-containing functional groups, while dodecane aggregates into spherical conformations on the surface of coal. Methyl laurate is easier to spread on the surface of coal. The head group (ester group) of methyl laurate covers the coal surface, while the tail group (fat chain) is exposed to the aqueous phase, forming a network structure with a better water barrier effect.

(2) Compared with dodecane, methyl laurate has a stronger interaction with the coal surface due to the existence of the ester group. The stronger interaction between methyl laurate and the coal surface makes it easier for methyl laurate to diffuse and adsorb on a sub-bituminous coal surface.

(3) The water molecule mobility of the two collectors on the modified coal surface follows the order of methyl laurate $>$ dodecane, which indicates that methyl laurate is a more effective collector to enhance the hydrophobicity of a low-rank coal surface than dodecane.

(4) XPS analysis showed that methyl laurate is an effective collector for reducing oxygen-containing functional groups on the surface of sub-bituminous coal compared with dodecane. The adhesion efficiency between air bubbles and (modified) coal particles was also measured. The results showed that the adhesion efficiency of sub-bituminous coal particles to bubbles increases after methyl laurate adsorption, which is higher than that of dodecane adsorption. This also means that after adding methyl laurate, coal particles are more easily attached to bubbles. The experimental results are in agreement with the simulation results, which verify the accuracy of the simulation.

Author Contributions: Data Acquisition, H.Z., W.L., H.X., Q.Z., Y.Z. and X.S.; methodology, H.Z., W.L., and H.X.; software, H.Z.; validation, H.Z., W.L., and H.X.; formal analysis, H.Z., W.L. and H.X..; writing一original draft preparation, H.Z.; writing-review and editing, H.Z., W.L., and H.X.; visualization, H.Z., W.L., and H.X.

Funding: This research was funded by the National Natural Science Foundation of China (NO. 51604280).

Conflicts of Interest: The authors declare no conflict of interest.

\section{References}

1. Willson, W.G.; Dan, W.; Irwinc, W. Overview of Low-Rank Coal (LRC) Drying. Coal Prep. 1997, 18, 1-15. [CrossRef]

2. Osman, H.; Jangam, S.V.; Lease, J.D.; Mujumdar, A.S. Drying of Low-Rank Coal (LRC)—A Review of Recent Patents and Innovations. Dry Technol. 2011, 29, 1763-1783. [CrossRef]

3. Xia, W.; Yang, J.; Liang, C. A short review of improvement in flotation of low rank/oxidized coals by pretreatments. Powder Technol. 2013, 237, 1-8. [CrossRef]

4. Jia, R.; Harris, G.H.; Fuerstenau, D.W. An improved class of universal collectors for the flotation of oxidized and/or low-rank coal. Int. J. Miner. Process. 2000, 58, 99-118. [CrossRef]

5. Wen, B.; Xia, W.; Sokolovic, J.M. Recent advances in effective collectors for enhancing the flotation of low rank/oxidized coals. Powder Technol. 2017, 319, 1-11. [CrossRef]

6. Dey, S. Enhancement in hydrophobicity of low rank coal by surfactants-A critical overview. Fuel Process Technol. 2012, 94, 151-158. [CrossRef]

7. Harris, G.H.; Diao, J.; Fuerstenau, D.W. Coal Flotation with Nonionic Surfactants. Coal Prep. 1995, 16, 135-147. [CrossRef]

8. Jena, M.S.; Biswal, S.K.; Rudramuniyappa, M.V. Study on flotation characteristics of oxidised Indian high ash sub-bituminous coal. Int. J. Miner. Process. 2008, 87, 42-50. [CrossRef]

9. Chen, S.; Tang, L.; Tao, X.; He, H.; Chen, L.; Yang, Z. Enhancing flotation performance of low rank coal by improving its hydrophobicity and the property of oily bubbles using 2-ethylhexanol. Int. J. Miner. Process. 2017, 167, 61-67. [CrossRef]

10. Vyas, A.P.; Verma, J.L.; Subrahmanyam, N. A review on FAME production processes. Fuel 2010, 89, 1-9. [CrossRef]

11. Xia, W. Biodiesel as a renewable collector for coal flotation in the future. Energy Sources 2016, 38, 1938-1943. [CrossRef]

12. Xia, W.; Yang, J.; Liang, C. Improving Oxidized Coal Flotation Using Biodiesel as a Collector. Coal Prep. 2013, 33, 181-187. [CrossRef] 
13. Xu, Y.; Liu, Y.L.; He, D.D.; Liu, G.S. Adsorption of cationic collectors and water on muscovite (001) surface: A molecular dynamics simulation study. Miner. Eng. 2013, 53, 101-107. [CrossRef]

14. Rai, B.; Sathish, P.; Tanwar, J.; Pradip; Moon, K.S.; Fuerstenau, D.W. A molecular dynamics study of the interaction of oleate and dodecylammonium chloride surfactants with complex aluminosilicate minerals. J. Colloid Interface Sci. 2011, 362, 510-516. [CrossRef] [PubMed]

15. Xia, Y.; Zhang, R.; Xing, Y.; Gui, X. Improving the adsorption of oily collector on the surface of low-rank coal during flotation using a cationic surfactant: An experimental and molecular dynamics simulation study. Fuel 2019, 235, 687-695. [CrossRef]

16. Xia, Y.; Yang, Z.; Zhang, R.; Xing, Y.; Gui, X. Enhancement of the surface hydrophobicity of low-rank coal by adsorbing DTAB: An experimental and molecular dynamics simulation study. Fuel 2019, 239, 145-152. [CrossRef]

17. Li, L.; Hao, H.; Yuan, Z.; Liu, J. Molecular dynamics simulation of siderite-hematite-quartz flotation with sodium oleate. Appl. Surf. Sci. 2017, 419, 557-563. [CrossRef]

18. Zhang, H.; Liu, W.; Han, C.; Wei, D. Intensify dodecylamine adsorption on magnesite and dolomite surfaces by monohydric alcohols. Appl. Surf. Sci. 2018, 444, 729-738. [CrossRef]

19. Zhang, Z.; Wang, C.; Yan, K. Adsorption of collectors on model surface of Wiser bituminous coal: A molecular dynamics simulation study. Miner. Eng. 2015, 79, 31-39. [CrossRef]

20. Guo, J.; Zhang, L.; Liu, S.; Li, B. Effects of hydrophilic groups of nonionic surfactants on the wettability of lignite surface: Molecular dynamics simulation and experimental study. Fuel 2018, 231, 449-457. [CrossRef]

21. Sun, H.; Jin, Z.; Yang, C.; Akkermans, R.L.; Robertson, S.H.; Spenley, N.A.; Miller, S.; Todd, S.M. COMPASS II: Extended coverage for polymer and drug-like molecule databases. J. Mol. Model. 2016, 22, 47. [CrossRef] [PubMed]

22. Hatcher, P.G. Chemical structural models for coalified wood (vitrinite) in low rank coal. Org. Geochem. 1990, 16, 959-968. [CrossRef]

23. Nose, S.A. A Unified formulation of the constant temperature molecular dynamics methods. J. Chem. Phys. 1984, 81, 511-519. [CrossRef]

24. Li, P.D. Investigation of Bituminous Coal Hydrophobicity and its Influence on Flotation. Energ. Fuel 2009, 23, 5536-5543.

25. Kozłowski, M. XPS study of reductively and non-reductively modified coals. Fuel 2004, 83, $259-265$. [CrossRef]

26. Zhuo, Q.; Liu, W.; Liu, W. Experimental study on the attachment behavior of coal particles and bubbles. J. Chin. Coal Soc. 2018, 43, 2029-2035.

27. You, X.; He, M.; Zhang, W.; Wei, H.; He, Q.; Lyu, X. Molecular dynamics simulations and contact angle of surfactant at the coal-water interface. Mol. Simul. 2018, 44, 1-6. [CrossRef]

28. He, M.; Zhang, W.; Cao, X.; You, X.; Li, L. Adsorption Behavior of Surfactant on Lignite Surface: A Comparative Experimental and Molecular Dynamics Simulation Study. Int. J. Mol. Sci. 2018, $19,437$. [CrossRef]

29. Tao, C.G.; Feng, H.J.; Zhou, J.; Lv, L.H.; Lu, X.H. Molecular Simulation of Oxygen Adsorption and Diffusion in Polypropylene. Acta Phys. -Chim. Sin. 2009, 25, 1373-1378.

30. Núñez-Rojas, E.; Domínguez, H. Computational studies on the behavior of sodium dodecyl sulfate (SDS) at $\mathrm{TiO} 2$ (rutile)/water interfaces. J. Colloid Interface Sci. 2011, 364, 417-427. [CrossRef]

31. Verrelli, D.I.; Koh, P.T.L.; Nguyen, A.V. Particle-bubble interaction and attachment in flotation. Chem. Eng. Sci. 2011, 66, 5910-5921. [CrossRef]

32. Lecrivain, G.; Petrucci, G.; Rudolph, M.; Hampel, U.; Yamamoto, R. Attachment of solid elongated particles on the surface of a stationary gas bubble. Int. J. Multiph. Flow 2015, 71, 83-93. [CrossRef]

33. Zhuo, Q.; Liu, W.; Xu, H.; Sun, X.; Zhang, H.; Liu, W. The Effect of Collision Angle on the Collision and Adhesion Behavior of Coal Particles and Bubbles. Processes 2018, 6, 218. [CrossRef]

(C) 2019 by the authors. Licensee MDPI, Basel, Switzerland. This article is an open access article distributed under the terms and conditions of the Creative Commons Attribution (CC BY) license (http:// creativecommons.org/licenses/by/4.0/). 